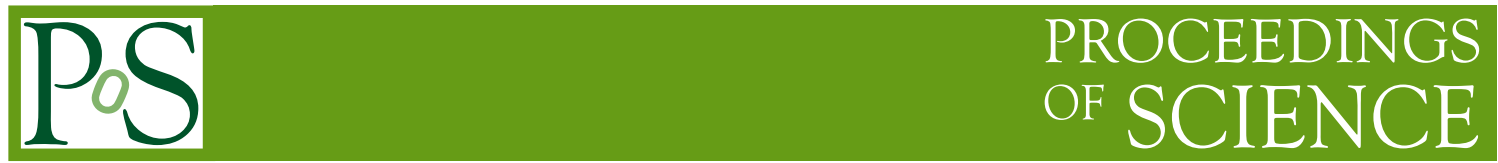

\title{
Recent developments in the tmLQCD software suite
}

\author{
A. Abdel-Rehim \\ CaSToRC, The Cyprus Institute, Nicosia, Cyprus \\ E-mail: a.abdel-Rehimecyi.ac.cy \\ F. Burger, B. Kostrzewa \\ Humboldt-Universität zu Berlin, Institut für Physik, Berlin, Germany \\ E-mail: florian.burger@hu-berlin.de, bartosz.kostrzewa@desy.de
}

\section{A. Deuzeman}

Albert Einstein Center for Fundamental Physics - University of Bern, Switzerland

E-mail: albert.deuzeman@gmail.com

\section{K. Jansen}

NIC, DESY, Zeuthen, Germany

E-mail: karl.jansen@desy.de

\section{Scorzato}

Trento Institute for Fundamental Physics and Application (TIFPA), Trento, Italy

E-mail: luigiescorzato.it

C. Urbach*

HISKP (Theory), Rheinische Friedrich-Wilhelms Universität Bonn, Germany

E-mail: urbach@hiskp.uni-bonn.de

We present an overview of recent developments in the tmLQCD software suite. We summarise the features of the code, including actions and operators implemented. In particular, we discuss the optimisation efforts for modern architectures using the Blue Gene/Q system as an example.

HU-EP-13/59,SFB/CPP-13-84

31st International Symposium on Lattice Field Theory - LATTICE 2013

July 29 - August 3, 2013

Mainz, Germany

\footnotetext{
${ }^{*}$ Speaker.
} 


\section{Introduction}

The Lattice QCD community relies to a large extent on the efficient usage of available computer resources. It is, therefore, mandatory to optimise existing codes for new supercomputer architectures as well as commodity systems newly appearing on the market.

Due to the increasing complexity and diversity of modern computer architectures there is need for flexible software which allows one to quickly implement and test new developments. Furthermore, lattice QCD actions nowadays simulated have also reached a high level of diversity, which needs to be mirrored by the software. Writing, debugging and implementing such software requires, hence, a non-negligible amount of manpower.

From this perspective it might appear sensible to develop only one, community wide code basis. However, for scientific hygiene there should be clearly at least two or three implementations available such that cross-checks are possible. Ideally, all the different implementations are publicly available, which increases the chance to find mistakes in the codes and allows any lattice QCD practitioner to re-use them (c.f. Ref. [1]).

By now there are several lattice QCD software suites available as open source, among others the MILC code [2], Chroma [3], openQCD [4] and bQCD [5]; another one is tmLQCD [6] obtained from github [7]. tmLQCD started as a code for simulations using the Wilson twisted mass formulation of lattice QCD, but includes by now a much wider range of actions and lattice Dirac operators. Moreover, tmLQCD is fully parallelised and includes optimisations for most modern supercomputer architectures.

\section{2. tmLQCD: General Overview}

The tmLQCD software is written in the $\mathrm{C}$ programming language following the C99 standard. It ships with an autoconf configuration script, which makes it relatively easy to compile the code on most modern computer platforms. It also comes with documentation as a LATEX document.

Once compiled, tmLQCD offers two executables: firstly an inverter offering a range of iterative solvers needed for computing propagators. The second executable implements a Hybrid Monte Carlo (HMC) algorithm [8] for generating gauge configurations using Wilson twisted mass actions, also including the clover term.

The various physical and algorithmic parameters of both programmes can by chosen by the user using an input file, which has a simple and human readable syntax. An example of the general section of such an input file for the HMC might look as follows:

$\begin{array}{ll}\mathrm{L}=4 & \# \text { spacial lattice extend } \\ \mathrm{T}=8 & \# \text { time extend } \\ \text { Measurements }=1000 & \# \text { no. of trajectories } \\ \text { StartCondition }=\text { hot } & \\ \text { ReversibilityCheck = yes } & \# \text { perform reversibility check } \\ \text { ReversibilityCheckIntervall=2 } & \# \text { every second traj. }\end{array}$

The code comes with a selection of sample input files. 
The executables can be compiled as scalar or parallel programmes to be decided at configure time. The parallelisation is implemented using a hybrid approach with the Message Passing Interface (MPI) and openMP. The MPI topology and the number of (openMP) threads per MPI task can be specified in the input file.

tmLQCD directly reads and writes the ILDG gauge configuration format [9] and the SCIDAC propagator format using the LIME library [10]. There is, therefore, full compatibility to the Chroma software. Moreover, tmLQCD can be configured to use the Lemon library [11], which is a parallelised replacement of LIME using the MPI parallel I/O capabilities. Lemon significantly increases the I/O performance on massively parallel machines. In particular, the propagator computation, usually heavily I/O bound, benefits from the usage of Lemon.

\section{Iterative Solvers}

One of the main tasks in lattice QCD is solving

$$
D \cdot \psi=\eta
$$

for $\psi$, where $D$ is some discretisation of the gauge covariant Dirac operator. Note that we have suppressed all indices for simplicity. The lattice Dirac operator can be viewed as a large sparse matrix, which makes iterative solvers and in particular Krylov space solvers like the conjugate gradient (CG) most suited for solving equation 3.1, see Ref. [12] for a general discussion.

Several discretisations of the Dirac operator are implemented in tmLQCD: the Wilson and Wilson twisted mass Dirac operators both with and without clover term, the non-degenerate Wilson twisted mass Dirac operator [13] with and without clover term and the overlap operator [14, 15]. For Wilson type operators also even/odd preconditioning [16] is implemented. The clover operators are currently only available with even/odd preconditioning. The operator can be specified in the input file like in the following example for a (mass degenerate) even/odd preconditioned Wilson twisted mass Dirac operator:

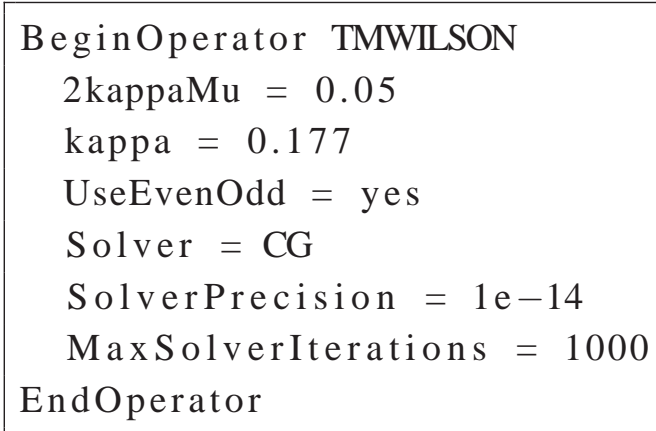

The physical parameters are the $\kappa=0.177$ hopping parameter and the twisted mass parameter $\mu$, the latter specified as $2 \kappa \mu=0.05$. In the above listing the iterative solver to be used is $C G$, the solver precision is set to $\|D \psi-\eta\|^{2} \leq 10^{-14}$ and not more than 1000 iterations should be used.

The list of available solvers includes CG, BiCG, BiCGstab, FGMRES, CGS, EigCG [17] and GCR. A FGMRES solver applying inexact deflation as discussed in Ref. [18] is also available, as well as a multiple mass CG solver for the twisted mass Dirac operator [19]. Note that the optimal (Krylov) solver depends on the particular discretisation of the lattice Dirac operator. 


\section{Lattice Actions}

Lattice QCD actions are usually split into a sum of a gauge and a fermionic part. Concerning the gauge part, tmLQCD implements the Wilson plaquette gauge action and the family of gauge actions including an additional planar $2 \times 1$ rectangular Wilson loop. These include the tree level Symanzik improved, the Iwasaki and the DBW2 gauge actions.

For the fermionic part we have implemented several so-called pseudofermion actions. They are based on the stochastic representation of a determinant of a matrix $Q^{2}$

$$
\operatorname{det}\left(Q^{2}\right)=\int \mathscr{D} \phi^{\dagger} \mathscr{D} \phi e^{-\phi^{\dagger} \frac{1}{Q^{2}} \phi},
$$

where the pseudofermion fields $\phi^{\dagger}, \phi$ follow bosonic statistics. Following the notation of Chroma, we call one such term a monomial and an example for an input file is as follows:

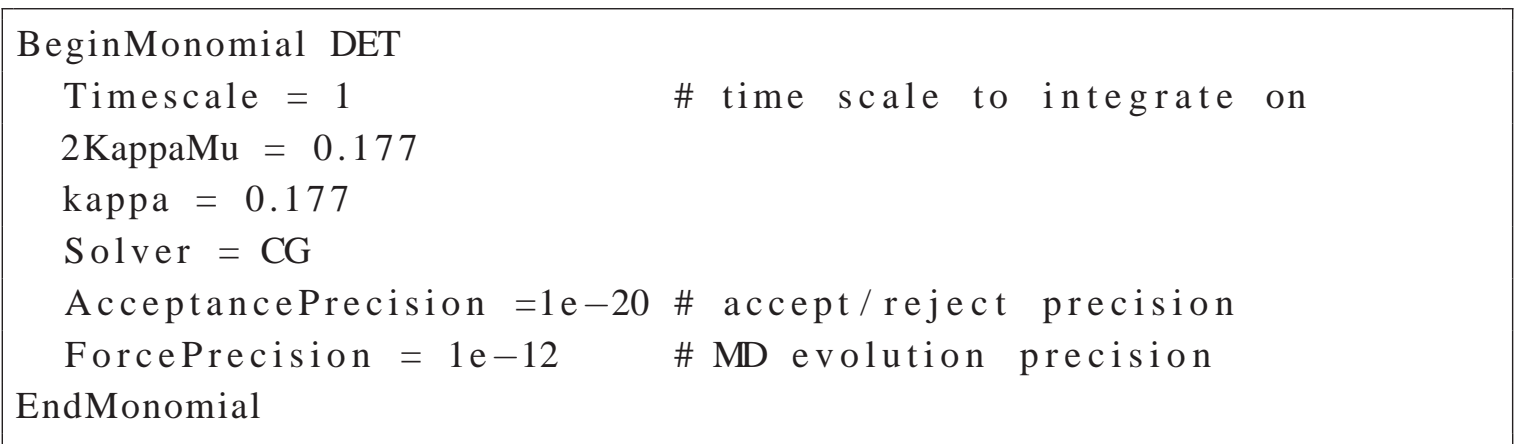

It corresponds to a two flavour, mass degenerate Wilson twisted mass Dirac operator pseudofermion monomial. You may specify the solver used in the HMC update, as well as the precisions for the molecular dynamics (MD) evolution and the accept/reject step.

There is a list of other monomials supported: for two mass degenerate Wilson and Wilson clover twisted mass fermions there are besides the "det" monomials also ratios of determinants needed for Hasenbusch mass preconditioning with multiple timescales [20, 21, 22]. For the mass non-degenerate Wilson and Wilson clover twisted mass doublet there is a polynomial [23] and a rational monomial [24] implemented. Finally, for a single Wilson clover fermion a rational monomial can be used.

tmLQCD offers different schemes for integrating the MD equations of motion: the simple leap-frog integration scheme, the second order minimal norm scheme and a fourth order Omelyan scheme $[25,26]$. They can be combined on different time scales, as can be seen from the following input file listing:

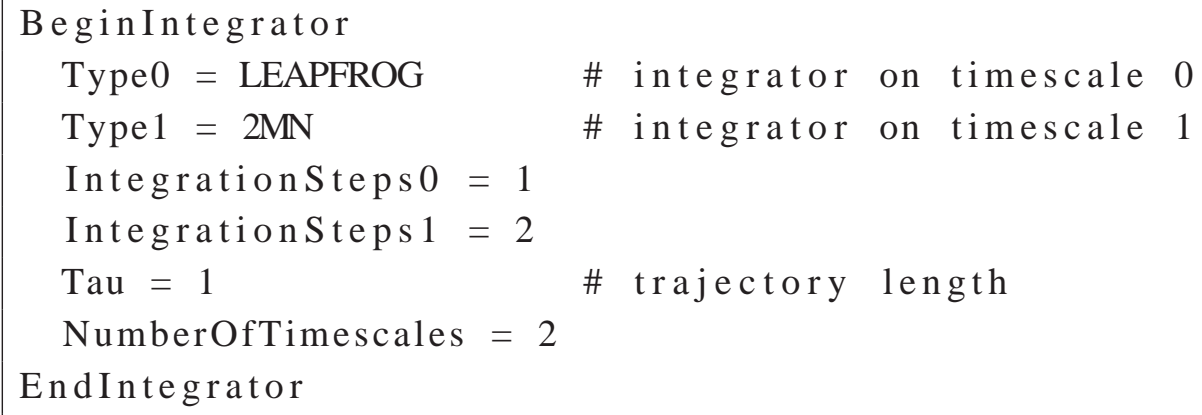


The zeroth time scale of the two is the finest, the total trajectory length is specified using $\tau=1$ and the number of steps for time scale $\mathrm{N}$ by IntegrationStepsN. The step numbers are defined recursively. Therefore, the step length on timescale zero is given by $\Delta \tau_{0}=\tau / N_{1} / N_{0}=\Delta \tau_{1} / N_{0}$, where $N_{i}$ is the number of steps on timescale $i$. Note that there are additional factors of $1 / 2$ for the higher order schemes.

\section{Optimisation: Example BG/Q}

As mentioned before, tmLQCD includes optimisations for several modern supercomputer architectures, IBMs Blue Gene/Q, Intels SSE instruction set and the Aurora architecture. We also have an inverter and parts of the HMC implemented for NVIDIA GPUs.

Here we will discuss as an example the BG/Q architecture. The BG/Q compute nodes consist of one CPU with 16 cores with four hardware threads each. Hence, in total there are 64 hardware threads per node, which can be divided into MPI tasks and/or (openMP) threads. The nodes are connected via a five dimensional torus network. For a first discussion on how to port lattice QCD codes for BG/Q see Ref. [27]. The floating point unit (FPU) includes a four double wide SIMD vector unit (QPX). For maximal performance it is mandatory to utilise it appropriately. In total one node of the BG/Q has a peak performance of $204.8 \mathrm{Gflop} / \mathrm{s}$.

For the following discussion we used a hybrid MPI/openMP implementation with always 64 openMP threads and one MPI task per BG/Q node. For a more detailed discussion on how to optimally use openMP see the contribution [28] at this conference. In figure 1 we show the performance of the tmLQCD Wilson Dirac hopping matrix in Gflop/s per BG/Q node as a function of the node local lattice extent $L_{\text {local }}$. Each node worked on a local lattice volume of $L_{\text {local }}^{4}$.

First we investigated the code performance without internode communication. The plain C99 implementation is shown as black diamonds leading to less than $5 \%$ of peak performance, almost independently of the local volume. This result points towards a badly saturated FPU. The red squares represent the code including the QPX instruction set utilising the intrinsic functions provided by the IBM C compiler. A strong improvement is visible, with up to $25 \%$ of peak at $L_{\text {local }}=12$. For $L_{\text {local }} \geq 14$ the local problem does no longer fit into the cache leading to a plateau in the performance around $20 \mathrm{Gflop} / \mathrm{s}$ per node.

The purple circles in figure 1 represent the hopping matrix with QPX instructions, but now with internode communication switched on. The MPI overhead turns out to be significant, more than halving the performance where the local volume fits into cache.

Circumventing this problem is possible by overlapping communication and computation. For this purpose we use an implementation of the hopping matrix where in a first step we project to half size spinors $\phi^{+}, \phi^{-}$for all $x$ and $\mu$

$$
\phi^{+}(x-\hat{\mu}, \mu)=U_{\mu}(x-\hat{\mu}) P_{\mu}^{(\text {full } \rightarrow \text { half })}\left(1-\gamma_{\mu}\right) \psi(x), \phi^{-}(x+\hat{\mu}, \mu)=P_{\mu}^{(\text {full } \rightarrow \text { half })}\left(1+\gamma_{\mu}\right) \psi(x) .
$$

In a second step the communication is performed and finally the result is generated by reconstructing the full spinor for all $x$

$$
\eta(x)=\sum_{\mu}\left[P_{\mu}^{(\text {half } \rightarrow \text { full })} \phi^{+}(x, \mu)+P_{\mu}^{(\text {half } \rightarrow \text { full })} U_{\mu}^{\dagger}(x-\hat{\mu}) \phi^{-}(x, \mu)\right] .
$$




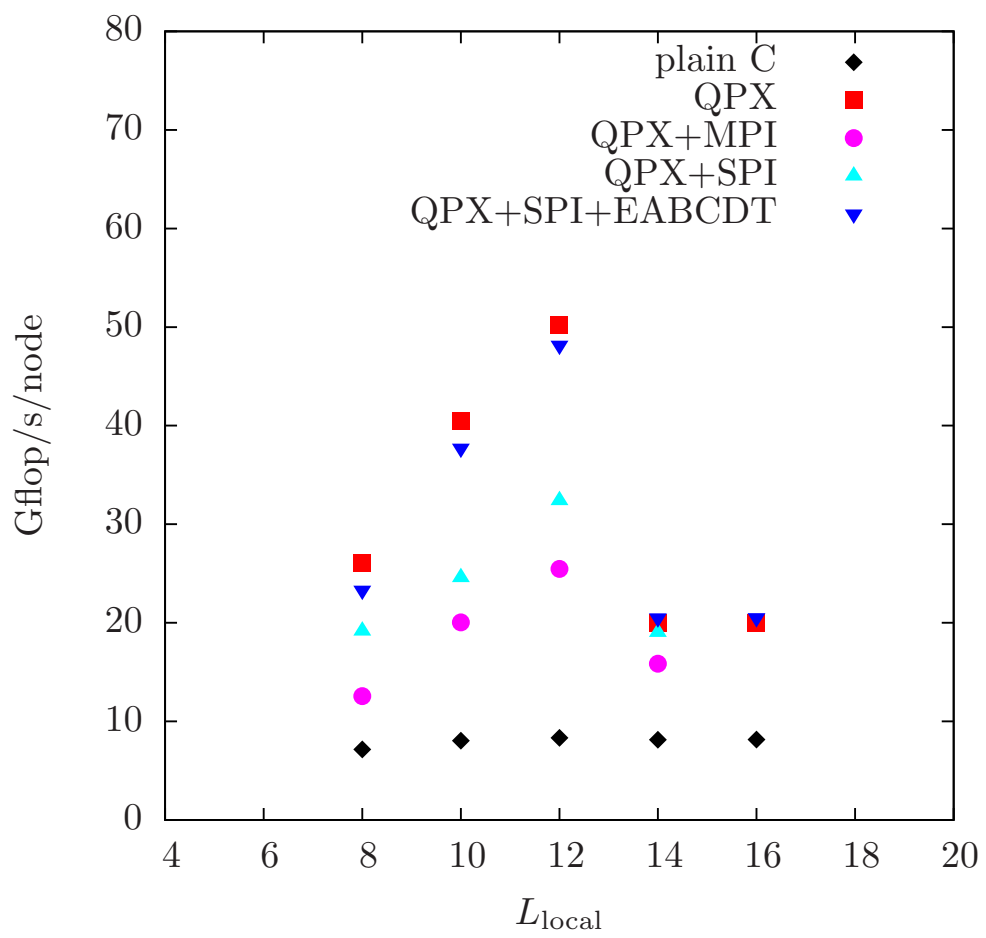

Figure 1: Single BG/Q node double precision performance of the hopping matrix in Gflop/s as a function of $L_{\text {local }}$. "Plain C" and "QPX" correspond to measurements with communication switched off using a plain C implementation and one including QPX instructions, respectively. The other points include communication, see text for details.

The field $\phi^{+,-}$requires only half the amount of data to be communicated compared to the full spinor field $\psi$. Overlapping communication and computation can now be achieved by dividing all $x$ into one set $x_{\text {surface }}$ residing on the local surface and a second set $x_{\text {bulk }}$. Then the projection to $\phi^{+,-}$is first done for all $x_{\text {surface, }}$ then the communication is started non-blocking in parallel to the the computation for $x_{\text {bulk. }}$. When the latter computation is finished it is checked whether the communication is finished also (c.f. also [29]).

It turns out that this does not work as expected using the MPI library provided on the BG/Q. All the communication is effectively done in the MPI_Wait and, therefore, there is no gain. However, IBM provides a lower level communication library called SPI. Replacing the MPI calls with SPI calls leads to the light blue triangles in figure 1 and, thus, to a significant improvement. The blue inverted triangles finally include also the correct mapping of the lattice QCD geometry to the five dimensional BG/Q network. So, eventually we obtain almost the same performance as in the case with communication switched off.

\section{Acknowledgements}

We thank all members of ETMC for the most enjoyable collaboration. B.K. is supported by the National Research Fund, Luxembourg. This work is supported in part by DFG and NSFC (CRC 110) and by DFG SFB/TR9. A. A.-R. acknowledges support from the PRACE-2IP project under grant number EC-RI-283493. LS thanks the SUMA project for partial support. 


\section{References}

[1] A. Deuzeman, PoS LATTICE2013 (2013).

[2] MILC Collaboration, http://www.physics.utah.edu/ detar/milc/.

[3] SciDAC, LHPC, UKQCD Collaboration, R. G. Edwards and B. Joo, Nucl.Phys.Proc.Suppl. 140, 832 (2005), arXiv:hep-lat/0409003 [hep-lat] .

[4] M. Lüscher, http://luscher.web.cern.ch/luscher/openQCD/.

[5] Y. Nakamura and H. Stüben, PoS LATTICE2010, 040 (2010), arXiv:1011.0199 [hep-lat ].

[6] K. Jansen and C. Urbach, Comput.Phys.Commun. 180, 2717 (2009), arXiv: 0905.3331 [hep-lat].

[7] ETM Collaboration, https://github.com/etmc/tmLQCD, 2013.

[8] S. Duane, A. Kennedy, B. Pendleton and D. Roweth, Phys.Lett. B195, 216 (1987).

[9] C. E. Detar, PoS LAT2007, 009 (2007), arXiv: 0710.1660 [hep-lat ].

[10] USQCD Collaboration, http://usqcd.jlab.org/usqcd-docs/c-lime/.

[11] A. Deuzeman, S. Reker and C. Urbach, Comput.Phys.Commun. 183, 1321 (2012), arXiv:1106.4177 [hep-lat].

[12] Y. Saad, Iterative Methods for sparse linear systems, 2nd ed. (SIAM, 2003).

[13] ALPHA Collaboration, R. Frezzotti, P. A. Grassi, S. Sint and P. Weisz, JHEP 08, 058 (2001), hep-lat/0101001.

[14] H. Neuberger, Phys.Lett. B417, 141 (1998), arXiv:hep-lat/9707022 [hep-lat].

[15] H. Neuberger, Phys.Lett. B427, 353 (1998), arXiv: hep-lat/9801031 [hep-lat].

[16] T. A. DeGrand and P. Rossi, Comput.Phys.Commun. 60, 211 (1990).

[17] A. Stathopoulos and K. Orginos, SIAM J.Sci.Comput. 32, 439 (2010), arXiv: 0707.0131 [hep-lat] .

[18] M. Lüscher, JHEP 0707, 081 (2007), arXiv: 0706.2298 [hep-lat ].

[19] T. Chiarappa et al., arXiv:hep-lat/0609023 [hep-lat].

[20] M. Hasenbusch, Phys.Lett. B519, 177 (2001), arXiv: hep-lat/0107019 [hep-lat].

[21] M. Hasenbusch and K. Jansen, Nucl.Phys. B659, 299 (2003), arXiv: hep-lat/ 0211042 [hep-lat].

[22] C. Urbach, K. Jansen, A. Shindler and U. Wenger, Comput.Phys.Commun. 174, 87 (2006), arXiv:hep-lat/0506011 [hep-lat].

[23] R. Frezzotti and K. Jansen, Nucl. Phys. B555, 395 (1999), hep-lat/ 9808011.

[24] M. A. Clark and A. D. Kennedy, hep-lat/0608015.

[25] J. Sexton and D. Weingarten, Nucl.Phys. B380, 665 (1992).

[26] I. P. Omelyan, I. M. Mryglod and R. Folk, Comput.Phys.Commun. 151, 272 (2003).

[27] P. Boyle, PoS LATTICE2012, 020 (2012).

[28] ETM Collaboration, B. Kostrzewa et al., PoS LATTICE2013, 416 (2013).

[29] M. Brambilla and F. Di Renzo, PoS LATTICE2012, 192 (2012). 